\title{
Use of creep recovery protocol to measure static yield stress and structural rebuilding of fresh cement pastes
}

\author{
Ye Qian and Shiho Kawashima*
}

Columbia University, Department of Civil Engineering and Engineering Mechanics, 500 West 120th street, New York, NY, 10027, USA

*Corresponding author at: Columbia University, Department of Civil Engineering and Engineering Mechanics, 500 West 120th street, 616 MUDD, New York, NY, 10027, USA.

Tel.: +1 2128542701.

Email address: s-kawashima@columbia.edu

\begin{abstract}
:
In this study, a creep recovery shear rheological protocolwas applied to fresh cement pastes. A viscosity bifurcation behavior was observed through applying a range of creep stresses. When applied stress is sufficiently low viscosity increases and the material yields, exhibiting viscoelastic solid-like behavior. Beyond a critical stress viscosity decreases and the material flows, exhibiting viscoelastic liquid-like behavior.Through examining this bifurcation behavior we found that the transition of viscosityoccurs at very low strains. Thestrains at which this transition occurred were compared with critical strainsmeasured through low amplitude oscillatoryshear. Results provided support thatthe solid-liquid transition occurs beyond the critical stress measured throughcreep, thereby tying it to static yield stress. The protocol was implemented to probepastes modified withattapulgite clays, a highly thixotropic system, and was found to be effective in characterizingstatic yield stress and thixotropic rebuilding.
\end{abstract}

Keywords:creep recovery test, static yield stress, viscosity bifurcation, solid-liquid transition, nanoclay. 


\section{Introduction}

Yield stress has been extensively studied in non-Newtonian fluids, includingcolloidal gel, bentonite,polymer suspension, food, paints, waxy crude oil,pastes, foams, etc[1-5]. Specifically in cementitious materials, yield stress is important in quantifying flowability[6] and is correlated to conventional field-friendly measurement methods such as slump flow and flow table test, both experimentally and in simulation[7]. The large application and research on self-consolidating concrete (SCC) has aroused great interest and study on yield stress because it is closely related to formwork pressure[8-14], stability of coarse particles in self-consolidating concrete (SCC)[15] and multi-layer casting[16].

Since the publication of the Bingham model[17], many models have been proposed to fit the equilibrium flow curve. The most common models forcementitious materials are Bingham, $\tau=\tau_{y}+\eta_{p l} \dot{\gamma}$, where $\tau$ is the shear stress, $\dot{\gamma}$ is the shear rate, $\tau_{\mathrm{y}}$ is the Binghamyield stress and $\eta_{\mathrm{pl}}$ is the plastic viscosity; and Herschel-Bulkley, $\tau=\tau_{y}+k \dot{\gamma}^{n}$, where $\mathrm{k}$ and $\mathrm{n}$ are constantsbased on material properties. Mortar and SCCareusually regarded as Bingham materials, showing a linear relationship between shear stress and shear rate[8, 18, 19]. While, because of shear thinning of cement paste[20]and shear thickening of normal concrete [21], cement paste and normal concrete are commonly fitted with the Herschel-Bulkley model, in which $\mathrm{n}<1$ for shear thinning and $n>1$ for shear thickening. With these models, the equilibrium flow curve quantifying the relationship between equilibrium shear stress and shear rate is fitted andthe dynamic yield stressisobtained. 
In contrast to the dynamic yield stress obtained from the steady-state flow curve, thixotropic materials also exhibit a static yield stress $[22,23]$. The dynamic yield stress is the stress necessary to terminate flow, measured after the material has been sheared and the microstructure has been broken down. In contrast, the static yield stress is the stress necessary to initiate flow, corresponding to a well-connected microstructure. A common protocol to characterize static yield stress is to apply an intermediate constant shear rate and to record the resultant shear stress[23, 24]. The typical response of cement-based materials is an initial increase to a peak and subsequent decrease to an equilibrium value over time. The increase in stress is related to the gradual deformation of the material and the peak stress marks the static yield stress of the suspension that must be overcome to initiate continuous flow. However, this method is shear rate dependent [25]. This presents an experimental challenge when attempting to characterize systems that exhibit very high rates of flocculation. For instance, systems modified with highly purified attapulgite clays were found to significantly accelerate thixotropic rebuilding kinetics[26]. As a result, the stress growth protocol is not a suitable approach to characterize such systems. Another method is to apply creep recovery, which was implemented by Struble and Schultz on cement paste systems[20]. This is a stress-controlled test, where the material will not flow until the applied creep stress is higher than the critical creep stress. They observed the transition from solid-like behavior to liquid-like behavior with a slight increase in applied stress. They compared the measured stress at which this transition occurs to the dynamic yield stress measured through fitting the flow curve with a steady-state flow model.Here, we adopt their approach and tie it to static yield stress, then extend it to characterize thixotropic rebuilding over time.

In this study, strain and viscosity development of cement paste under a creep recovery test will be presented and discussed. Through incrementally increasing applied stress, a viscosity bifurcation is found to occurbeyonda critical stress. Through low amplitude oscillatory shear, the critical 
strain at which the solid-liquid transition occurs is measured and is compared to the shear strains measured in the creep recovery test. The strains at which the material transitions to either flow or no flow werefound to exceed the critical strain only once the critical stress had been exceeded.This supports that the critical stress measured through the creep recovery test is static

yield stress. The testis extended to characterize thixotropic rebuilding of cement pastes modified with attapulgite clays by measuring the development of static yield stress over time, up to $60 \mathrm{~min}$.

\section{Theoretical background of approach}

\subsection{Creep recovery test}

Creep recovery test has long been applied to probe the viscoelastic properties of materials. The protocol and various viscoelastic responses are shown in Figure 1. A shear stress is applied instantaneously to the material, held constant for a period of time, and then removed to (Figure 1a)[20, 27].For an elastic solid (Figure 1b) the load-deformation relationship is proportional, where the strain changes instantaneously with stress and stays constant; when the stress is removed the strain instantaneously drops to 0 and stays constant. For a viscous liquid(Figure 1c) the load-deformation rate relationship is proportional, where the strain linearly increases with time with no initial strain;when the stress is removedthere is no recovery and the strain remains constant. For viscoelastic materials, there is an instantaneous strain,which is then followed by a time-dependent strain. For viscoelastic solids the strain reaches an equilibrium value (Figure 1d) while for viscoelastic liquid the strain continues to increase (Figure 1e).Upon removal of stress, viscoelastic solidscan recovery substantially while viscoelastic fluidsexhibit little to no recovery. 
Many researchers have found that under different stresses, fresh cement-based materials show different strain development. Struble et al.[20] found that under low shear stresses cement pastes show a creep recovery response similar to viscoelastic solids, with strain reaching equilibrium under creep and partial recovery of strain upon removal of stress. Under higher shear stressescement pastes show a similar response to viscoelastic liquids, where strain continues to increase under creep and exhibits no recovery. Struble et al. reasoned that the solid-liquid transition occurs within a narrow range of shear stresses and can be linked to the yield stress of the material.

Fresh cementitious materials are thixotropic, exhibiting flocculation due to diffusion/coagulation processesand formation of CSH bridges due to early hydration under rest(structural rebuilding) and deflocculationand breakage of CSH bridges under shear (shear rejuvenation)[28]. With respect to macroscale flow, this translates to increase in viscosity under rest and decrease under shear. Using the inclined plane test, Coussot et. al. [29, 30]observed avalanche behavior in yield stress fluids. There exists a critical slope, which corresponds to a critical stress applied on the fluid. Within the critical slope the fluid exhibits little to no flow, while with only a slight increase in slope the fluid flows much longer and faster. It is believed to be the effect of thixotropy and the competition between structural rebuilding and shear rejuvenation. When stress is not sufficiently highstructural rebuilding is dominant over shear rejuvenation, thus the flow stops eventually; while with slightly higher stress shear rejuvenation becomes dominant and the material exhibits continuous flow that accelerates until steady-state is reached. In this study, it is proposed that the creep recovery protocolcan be implemented to observe similar behavior through viscosity bifurcation and subsequently used to characterize static yield stress. 


\subsection{Oscillatory test}

The oscillatory shear approach has been largely applied to study the viscoelastic properties of fresh cement pastes. Low amplitude oscillatory shear (LAOS) can be applied to obtain the evolution of storage and loss modulus [31]. And amplitude sweeps can be applied to obtain measures of critical strain[32]. The theory is briefly explained here.

An oscillatory strain is applied as a sine function:

$$
\gamma=\gamma_{0} \sin (\omega t)=\gamma_{0} \sin (2 \pi f t) \quad \text { Eq. } 1
$$

where $\gamma_{0}$ is maximum strain amplitude $[\mathrm{rad}]$, $\mathrm{t}$ is time $[\mathrm{s}]$, and $\omega$ is angular frequency $[\mathrm{rad} / \mathrm{s}]$ and $\mathrm{f}$ is frequency $[\mathrm{Hz}]$. If the strain is sufficiently low so that the particles in the suspension remain close to each other, the microstructure is not disturbed and the material can recover elastically. In this case (linear regime), the measured response in terms of stress is as follows:

$$
\tau=\gamma_{0}\left[G^{\prime} \sin (\omega t)+G^{\prime \prime} \cos (\omega t)\right] \quad \text { Eq. } 2
$$

where $G^{\prime}$ is the storage modulus, and G' is the loss modulus. The storage modulus represents the elastic or in-phase component and the loss modulus represents the viscous or out-of-phase component. For an ideal elastic solid, G” equals 0 ; and for an ideal fluid, G' equals 0.

The first step in performing oscillatory shear rheometery is to find the linear viscoelastic region (LVR), where G' (and also G”) is independent of applied frequency and strain. For a flocculated suspension, particles are able to recover elastically as strain is oscillated until some critical strain 
amplitude is reached. Within this critical strain, which is the LVR, strainsare sufficiently small sothat the structural integrity of the flocculated network is maintained. Above the critical strain, beyond the LVR, strains are sufficiently high so that particles are not able to recover elastically, modulus decreases, and the material behaves as a viscoelastic liquid.

To find the LVR a strain sweep is performed on cement pastes, during which the sample is subjected to an oscillatory strain of constant frequency and increasing amplitude, which may range in the order of 5 or 6 . The corresponding stiffnessG' curve will exhibit two main branches, as shown inFigure 2: a plateau region corresponding to the LVR and a decreasing branch indicating that the sample is experiencing a shear-induced breakage. The strain at which there is an apparent drop of stiffness marks the end of the LVR and is considered to be the critical strain. Frequency must also be considered to find the LVR. If the frequency is too high, the structure cannot recover and the measured LVR will be inaccurate.

Here, we will implement the amplitude strain sweep to measure critical strain, at which the transition from viscoelastic solid to viscoelastic liquid occurs. This will serve to supplement the results of the creep test to verify that the stress measured can be tied to static yield stress. Critical strain can also provide some insight into the fresh-state microstructure. Therefore the effect of attapulgite clay addition on critical strain will be measured.

\section{Material and procedures}

\subsection{Materials and experimental setup}


Type I Portland cement is used in all mixes. According to ASTM C150 [33], its compressive strength at 28 days is $44.8 \mathrm{MPa}$, the Blaine fineness is $420 \mathrm{~m}^{2} / \mathrm{kg}$ and the chemical constituents are summarized in Table 1. Mixes are prepared with tap water. Highly purified attapulgite clay is also added to select mixes. The nanoclay is a highly purified magnesium alumino-silicate. It is a commercially available clay that is chemically exfoliated from bulk attapulgite to remove all impurities. When dispersed, it is needle-like with an average length of $1.75 \mu \mathrm{m}$ and diameter of 3 $\mathrm{nm}$, so it will be referred to as nanoclay herein. In this study, no chemical admixtures are used.

Mixeshave a water-to-cement ratio (W/C) of 0.36 by mass. As a parameter, the amount of nanoclay varies from $0,0.1,0.3$ and $0.5 \%$ by mass of cement.

Nanoclay powder is blended with the mixing water in a Waring blender for 2 minutes to produce a clay suspension, which remains stable after 6 hours at rest. Immediately after preparing the suspension, it is poured into a beaker. Cement powder is slowly added to the suspension and mixed by hand for $1 \mathrm{~min}$, then mixed with a hand mixer at a speed of $1100 \mathrm{rpm}$ for $3 \mathrm{~min}$. The fresh cement paste is loaded into the cup of therheometer with a syringe to ensure each sample is the same volume.

We perform all tests on a HAAKE MARS III rotational rheometer. We use a coaxial cylinder geometer - the radius of the bob is $12.54 \mathrm{~mm}$ and the gap between the bob and cup is $1.06 \mathrm{~mm}$.

\subsection{Shear rheological protocols}


As cementitious materialsare thixotropic, the flow behavior is greatly dependent on the flocculation state. It must be consistent before each test to guarantee repeatability. For all protocols in this study, each sample is presheared at $600 \mathrm{~s}^{-1}$ for 4 min until the shear stress reaches equilibrium,after which the material is allowed to rest for a period of time for stress relaxationand to reach a stable state.Then, either the creep recovery test or oscillatory test is applied. In the creep recovery protocol, we apply the creep step for $60 \mathrm{sec}$ at each applied stress level and monitor its recovery for up to $60 \mathrm{sec}$. In the strain amplitude sweep test, we apply strains from $10^{-}$ ${ }^{5}$ to $10^{-1}$ at a fixed frequency of $1 \mathrm{~Hz}$, as conducted in many studies on cement paste $[31,34,35]$.

\section{Results and discussion}

\subsection{Creep-recovery - viscosity bifurcation}

We implement the creep-recovery protocol on neat cement pastes.Figure 3 and Figure 4 show the strain and viscositydevelopment under various creep stresses, respectively.As shown in Figure 3a, when creep stress is below $18.9 \mathrm{~Pa}$ the strain reaches a plateau and flow ceases. When creep stress is higher than $19 \mathrm{~Pa}$ the strain increases and the material continues to flowuntil creep stress is retrieved. Supplementing this with the recovery,Figure 3b, it is observed that within $18.9 \mathrm{~Pa}$ the material exhibits some recovery (viscoelastic solid) and beyond $19 \mathrm{~Pa}$ there is no recovery (viscoelastic fluid). 
As could be seen in Figure 4, under creep the viscosity exhibits an initial plateau and then either increases or decreases. When creep stress is below 18.9 Pa the viscosity increases to infinite values; when higher than $19 \mathrm{~Pa}$ the viscosity decreases to diminishingly small values. This resembles the avalanche behavior of thixotropic materials, as discussed prior.Thus this bifurcation point can be tied to the transition between viscoelastic solid to liquid, and the corresponding critical stress may be considered to be the static yield stress. It can be seen that the transition occurs at the very early ages of creep. Under stresses higher than the static yield stress, the material transitions from a solid-like structure to a liquid-like structure. It is also assumed that this transition is related to the microstructure of material. This is explored through LAOS and discussed in the next section.

\subsection{Solid-liquid transition}

\subsubsection{Amplitude sweep - critical strain}

We implement amplitude and frequency sweeps on fresh cement pastes. Through monitoring G', we measure the LVR and corresponding critical strain.A frequency sweep experiment is performed in which frequencies from $0.1 \mathrm{~Hz}$ to $20 \mathrm{~Hz}$ are applied at a fixed strain of $10^{-4}$. Results of frequency sweeps indicated that $1 \mathrm{~Hz}$ is within the LVR, which is also commonly applied in other studies $[31,35,36]$. Therefore this fixed frequency is used for all amplitude sweeps. The

results of an amplitude sweep on neat paste is shown in Figure 5, where strains ranging from $10^{-5}$ to 0.1 are applied. Beyond a critical strain, storage modulusG' decreases dramatically, showing that the structure is broken down. In addition, beyond this point G'becomes greater than G', indicating the transition from solid-like to liquid-like behavior. The average value of the critical 
strain measured through low amplitude oscillatory sweep of neat paste is $7.69 \times 10^{-4}$, which corresponds to the same order of magnitude as those reported in other works[31, 37].

\subsubsection{Solid-liquid transition}

The transition strain is recorded from viscosity evolution plots (Figure 4), considered to bewhen the viscosity becomes $20 \%$ higher or lower than the initial plateau value, at each creep stress. Transition trainis plotted against applied stressand presented inFigure 6 . In addition, these values are compared againstthe critical strain as measured by LAOSand critical stressas measured by creep protocol, indicated by the dotted lines. With increasing creep stress, the transition strain increases, as shown inFigure 6a. It could be seen that under creep stresses lower than the critical stress the transition strain remains within the critical strain, implying that the microstructure remains intact, as shown in Figure 6b. Further, under creep stresses higher thanthe critical stress the transition strain exceedsthe critical strain, indicating the microstructure is no longer able to recovery elastically and the solid-liquid transition occurs. Only when applied creep stress exceedsthe static yield stress could the material show solid-liquid transition. This will be discussed further in the following subsection.

\subsubsection{Schematic diagram of creep behavior}

Baudez and Coussot[38] found that under creep in a couette geometry, typical yield stress materials (i.e. Carbopol gel and foam)initially deformedhomogeneously .Then beyond a critical deformation the inner rotor region deformed much more quickly than the region near the wall, 
indicating solid-liquid transition occurred near the rotor region.Although Carbopol gel and foams have much higher critical strains than cement pastes, they still show very similar yield stress and viscoelastic properties. The authors believe that before the transition between flow and no flow, the viscosity at the plateau in Figure 4is the instantaneous response of the viscoelastic solid material. At this point, the transition has not yet occurred, so the material is homogeneously deformed. And the higher the creep stress, the higher the transition strain.

After the transition, the bifurcation behavior is determined by whether the strain exceeds the critical strain. As illustrated inFigure 7, with increasing stress the transition strain increases. For creep stresseswithinthe static yield stress, the transition strain is smaller than the critical strain. The structure is not broken so viscosity approaches infinity and flow ceases. In contrast, for creep stressesexceeding the static yield stress the transition strain is higher than the critical strain. The structure is broken, the flow continues and the viscosity decreases due to shear rejuvenation. The agreement between the oscillatory amplitude sweep and creep-recovery tests provides support that the critical stress measured through the creep protocol is static yield stress.

\subsection{Effect of nanoclay}

\subsubsection{Static yield stress}

As mentioned prior, due to the rate dependence of the stress growth protocol in measuring static yield stress it is not a suitable approach for characterizing highly thixotropic mixes. Therefore here we implement the creep-recovery protocol to measure the static yield stress of cement pastes 
modified with highly purified attapulgite clays, which exhibit high rate of thixotropic rebuilding at short time scales[26].The results of static yield stress as measured by the creep-recovery protocol on cement pastes with nanoclay addition are shown inFigure 8. It isshown that there is a linear increase of static yield stress with nanoclay addition. With $0.5 \%$ of nanoclay addition over cement, the static yield stress increases 3 times. This is in good agreement with the results found that measured the effect of these nanoclays on cohesive strength as measured by the tack test[37].

It is considered that[16] of the two components of thixotropy, i.e. de-flocculation under shear and flocculation at rest, the latter is more important because most of the problems encountered in SCC application (i.e. formwork pressure, multi-layer casting, and stability) are all related to resting. The accurate quantification of static yield stress is the key to solving these problems. $\mathrm{A}_{\text {thix }}$ quantified as the increment of yield stress over resting time, shown in Eq. 3, has been proposed as a useful parameter to describe the thixotropy of materials[16].

$$
\tau_{0}(t)=\tau_{0}+A_{\text {thix }} t \quad \text { Eq. } 3
$$

Nanoclay has been found to effectively decrease formwork pressure in lab-scale studies[39]. And this has been attributed to its effect on thixotropic rebuilding over time. Therefore we measured the effect of nanoclay on static yield stress over time. The results are shown inFigure 9. It is apparent that with increasing resting time, the static yield stress of both cement paste with and without nanoclay increase. From 1 to $30 \mathrm{~min}$, the thixotropic rate $\mathrm{A}_{\text {thix }}$ of cement pastewith 0 and $0.5 \%$ nanoclayare $2.35 \mathrm{~Pa} / \mathrm{min}$ and $6.61 \mathrm{~Pa} / \mathrm{min}$, respectively. From 30 to $60 \mathrm{~min}, \mathrm{~A}_{\text {thix }}$ of cement pastewith 0 and $0.5 \%$ nanoclayare $5.43 \mathrm{~Pa} / \mathrm{min}$ and $11.25 \mathrm{~Pa} / \mathrm{min}$, respectively. With addition of nanoclay, the cement paste exhibits higher rate of thixotropic rebuilding. As modeled by Ovarlez and Roussel[11], the higher the $\mathrm{A}_{\text {thix }}$, the lower the formwork pressure. Thus, this effect on static 
yield stress with resting time by the nanoclays can help partially explain the substantial decrease in formwork pressure over time measured in previous studies[39].

$\mathrm{A}_{\text {thix }}$ also increases with longer resting time. From the first $30 \mathrm{~min}$ to the second $30 \mathrm{~min}$ of resting, the $\mathrm{A}_{\text {thix }}$ of cement paste without nanoclay increases 2.31 times, while it increases 1.70 time with $0.5 \%$ of nanoclay. A potential explanation may be tied to cement hydration. Studies by Kawashima [26] showed that after 30 min of resting, storage modulus of cement pastes without nanoclay becomes higher than those with $0.5 \%$ nanoclay, implying that the effect of nanoclays on thixotropic rebuilding may be hindered by the progression of hydration. This is currently under investigation.

\subsubsection{Critical strain}

The yield stress of cement paste originates from the microstructure of an attractive particleparticle network, connected through colloidal interaction or direct contact. The microstructure sustains a certain amount of stress before it is broken and starts to flow, which defines the yield stress. Other studies have measured the clay's effect on floc size and strength. However, characterization was limited to steady-state shear conditions[40, 41]. To gain insight into how the attapulgite clays affect the undisturbed fresh-state microstructure at rest, we measure the critical strain.

Results ofLAOSon nanoclay-modified cement pastes are shown in Figure 10. They indicate that critical shear strain decreases with nanoclay addition. Due to their fine size compared to cement 
particles - nanoclays are $1.75 \mu \mathrm{m}$ in length, $3 \mathrm{~nm}$ in diameter while Ordinary Portland Cement has an average particle size of $15 \mu \mathrm{m}$ - we may attribute this to the clays filling the voids between cement particles and connecting the bonds between cement particles; or due to surface and edge charge effects nanoclay itself also forms card-house type structures, contributing to the connectivity of the cement paste microstructure.In section 4.2.2 it was discussed how the critical strain measured by LAOS agreed well with the solid-liquid transition measured by creep. The authors would like to point out that similar behaviour was found for all pastes modified withnanoclay addition, i.e. at $0.1,0.3$ and $0.5 \%$, tested in this study. So the theory described by Figure 7 also applies to pastes systems with nanoclay.

\section{Conclusions}

The creep recovery test is applied to probe the viscoelastic behavior of cement paste and a viscosity bifurcation is observed. Within a critical stress the strain approaches equilibrium, indicating no flow. Beyond a critical stressthe strain increases dramatically and no equilibrium value is reached, indicating flow is initiated and continues. The critical stress corresponding to this viscosity bifurcation is considered to mark the solid-liquid transition of the material and so is considered to be a measure of static yield stress. Measures of critical strain by LAOS are found to support this. The methods are applied to characterize the thixotropic rebuilding of cement pastes modified with nanoclays up to $0.5 \%$ by mass of cement. The creep protocol is found to effectively capture the static yield stress of highly thixotropic systems. Further, the increase in static yield stress over resting time is found to be higher for cement paste with $0.5 \%$ nanoclay over neat paste. This helps to elucidate how nanoclays can reduce formwork pressure over time. 
Nanoclaysare also found to decrease critical strain measured by LAOS, which provides evidence that they are increasing the interconnectivity of the fresh-state microstructure.

\section{Acknowledgements}

The authors would like to acknowledge the National Science Foundation (NSF EEC-1342377) and Columbia University's Office of the Provost's Grants Program for financial support, and technical support by the staff of Columbia University's Carleton Laboratory. Also, the authors would like to thank Essex Cement LLC and Active Minerals for providing materials. 


\section{Reference}

[1] H. Barnes, K. Walters, The yield stress myth?, Rheol Acta, 24 (1985) 323-326.

[2] H.A. Barnes, The'Yield Stress Myth?'Paper--21 Years On, Applied Rheology, 17 (2007) $43110-44250$.

[3] H.A. Barnes, The yield stress - a review or ' $\pi \alpha \nu \tau \alpha \rho \varepsilon \imath$ - everything flows?, Journal of NonNewtonian Fluid Mechanics, 81 (1999) 133-178.

[4] J.P. Hartnett, R.Y. Hu, Technical note: the yield stress-an engineering reality, Journal of Rheology (1978-present), 33 (1989) 671-679.

[5] P. Moller, A. Fall, V. Chikkadi, D. Derks, D. Bonn, An attempt to categorize yield stress fluid behaviour, Philosophical Transactions of the Royal Society of London A: Mathematical, Physical and Engineering Sciences, 367 (2009) 5139-5155.

[6] C.F. Ferraris, Measurement of the rheological properties of high performance concrete: state of the art report, JOURNAL OF RESEARCH-NATIONAL INSTITUTE OF STANDARDS AND TECHNOLOGY, 104 (1999) 461-478.

[7] V. Mechtcherine, A. Gram, K. Krenzer, J.-H. Schwabe, S. Shyshko, N. Roussel, Simulation of fresh concrete flow using Discrete Element Method (DEM): theory and applications, Mater Struct, 47 (2014) 615-630.

[8] J. Assaad, K.H. Khayat, H. Mesbah, Assessment of thixotropy of flowable and selfconsolidating concrete, ACI Materials Journal, 100 (2003).

[9] J. Assaad, K.H. Khayat, Variations of lateral and pore water pressure of self-consolidating concrete at early age, ACI Materials Journal, 101 (2004).

[10] K. Khayat, J. Assaad, H. Mesbah, M. Lessard, Effect of section width and casting rate on variations of formwork pressure of self-consolidating concrete, Mater Struct, 38 (2005) 73-78. 
[11] G. Ovarlez, N. Roussel, A physical model for the prediction of lateral stress exerted by selfcompacting concrete on formwork, Mater Struct, 39 (2006) 269-279.

[12] J. Tchamba, S. Amziane, G. Ovarlez, N. Roussel, Lateral stress exerted by fresh cement paste on formwork: laboratory experiments, Cement and Concrete Research, 38 (2008) 459-466.

[13] S. Kawashima, J.H. Kim, D.J. Corr, S.P. Shah, Study of the mechanisms underlying the fresh-state response of cementitious materials modified with nanoclays, Construction and Building Materials, 36 (2012) 749-757.

[14] P. Billberg, Form pressure generated by self-compacting concrete: influence of thixotropy and structural behaviour at rest, (2006).

[15] N. Roussel, A theoretical frame to study stability of fresh concrete, Mater Struct, 39 (2006) $81-91$.

[16] N. Roussel, A thixotropy model for fresh fluid concretes: theory, validation and applications, Cement and Concrete Research, 36 (2006) 1797-1806.

[17] E.C. Bingham, Fluidity and plasticity, McGraw-Hill Book Compny, Incorporated,, 1922.

[18] J. Assaad, K.H. Khayat, Assessment of Thixotropy of Self-Consolidating Concrete and Concrete-Equivalent-Mortar- Effect of Binder Composition and Content, ACI Materials Journal, $101(2004)$.

[19] J.J. Assaad, K.H. Khayat, Effect of viscosity-enhancing admixtures on formwork pressure and thixotropy of self-consolidating concrete, ACI Materials Journal, 103 (2006).

[20] L.J. Struble, M.A. Schultz, Using creep and recovery to study flow behavior of fresh cement paste, Cement and concrete research, 23 (1993) 1369-1379.

[21] F. De Larrard, C. Ferraris, T. Sedran, Fresh concrete: a Herschel-Bulkley material, Mater Struct, 31 (1998) 494-498.

[22] D.C. Cheng, Yield stress: a time-dependent property and how to measure it, Rheol Acta, 25 (1986) 542-554. 
[23] F. Mahaut, S. Mokeddem, X. Chateau, N. Roussel, G. Ovarlez, Effect of coarse particle volume fraction on the yield stress and thixotropy of cementitious materials, Cement and concrete research, 38 (2008) 1276-1285.

[24] G. Ovarlez, X. Chateau, Influence of shear stress applied during flow stoppage and rest period on the mechanical properties of thixotropic suspensions, Physical Review E, 77 (2008) 061403.

[25] A.W. Saak, H.M. Jennings, S.P. Shah, The influence of wall slip on yield stress and viscoelastic measurements of cement paste, Cement and concrete research, 31 (2001) 205-212.

[26] S. Kawashima, M. Chaouche, D.J. Corr, S.P. Shah, Rate of thixotropic rebuilding of cement pastes modified with highly purified attapulgite clays, Cement and Concrete Research, 53 (2013) 112-118.

[27] A. Zosel, Rheological properties of disperse systems at low shear stresses, Rheol Acta, 21 (1982) 72-80.

[28] N. Roussel, G. Ovarlez, S. Garrault, C. Brumaud, The origins of thixotropy of fresh cement pastes, Cement and Concrete Research, 42 (2012) 148-157.

[29] P. Coussot, Q.D. Nguyen, H. Huynh, D. Bonn, Avalanche behavior in yield stress fluids, Physical review letters, 88 (2002) 175501.

[30] P. Coussot, S. Boyer, Determination of yield stress fluid behaviour from inclined plane test, Rheol Acta, 34 (1995) 534-543.

[31] M.A. Schultz, L.J. Struble, Use of oscillatory shear to study flow behavior of fresh cement paste, Cement and Concrete Research, 23 (1993) 273-282.

[32] N. Roussel, A. Lemaître, R.J. Flatt, P. Coussot, Steady state flow of cement suspensions: a micromechanical state of the art, Cement and Concrete Research, 40 (2010) 77-84.

[33] ASTM C150, Standard Specification for Portland Cement, in, ASTM International, West Conshohocken, PA, 2015. 
[34] L. Nachbaur, J. Mutin, A. Nonat, L. Choplin, Dynamic mode rheology of cement and tricalcium silicate pastes from mixing to setting, Cement and Concrete Research, 31 (2001) 183192.

[35] A. Betioli, P. Gleize, D. Silva, V. John, R. Pileggi, Effect of HMEC on the consolidation of cement pastes: Isothermal calorimetry versus oscillatory rheometry, Cement and concrete Research, 39 (2009) 440-445.

[36] Z. Sun, T. Voigt, S.P. Shah, Rheometric and ultrasonic investigations of viscoelastic properties of fresh Portland cement pastes, Cement and Concrete Research, 36 (2006) 278-287.

[37] S. Kawashima, M. Chaouche, D.J. Corr, S.P. Shah, Influence of purified attapulgite clays on the adhesive properties of cement pastes as measured by the tack test, Cement and Concrete Composites, (2014).

[38] J. Baudez, P. Coussot, Abrupt transition from viscoelastic solidlike to liquidlike behavior in jammed materials, Physical review letters, 93 (2004) 128302.

[39] J.H. Kim, M. Beacraft, S.P. Shah, Effect of mineral admixtures on formwork pressure of self-consolidating concrete, Cement and Concrete Composites, 32 (2010) 665-671.

[40] R.D. Ferron, S. Shah, E. Fuente, C. Negro, Aggregation and breakage kinetics of fresh cement paste, Cement and Concrete Research, 50 (2013) 1-10.

[41] N.A. Tregger, M.E. Pakula, S.P. Shah, Influence of clays on the rheology of cement pastes, Cement and concrete research, 40 (2010) 384-391. 


\section{List of Tables}

Table 1 Cement chemical constituents

\begin{tabular}{cc}
\hline Constituents & \% by mass \\
\hline $\mathrm{SiO}_{2}$ & 19.22 \\
$\mathrm{Al}_{2} \mathrm{O}_{3}$ & 4.98 \\
$\mathrm{Fe}_{2} \mathrm{O}_{3}$ & 3.42 \\
$\mathrm{CaO}$ & 62.42 \\
$\mathrm{MgO}$ & 3.87 \\
$\mathrm{SO}_{3}$ & 2.72 \\
\hline
\end{tabular}

\section{List of Figures}

Figure 1 (a) Applied creep recovery protocol, and strain development of (b) elastic solid, (c) viscous liquid, (d) viscoelastic solid and (e) viscoelastic liquid.

Figure 2 Critical strain measured through low amplitude oscillatory strain sweep.

Figure 3 Strain development of cement paste under (a) creep and (b) recovery.

Figure 4 Viscosity bifurcation of cement paste.

Figure 5 Strain sweep of cement paste with w/c at 0.36 .

Figure 6 Transition strain versus applied creep stress, in comparison with critical stress and critical strain - (a) Full and (b) partial plot.

Figure 7 Schematic depicting the comparison between transition strain (creep) and critical strain (LAOS) - different applied stress leads to different flow behavior.

Figure 8 Effect of nanoclay addition on static yield stress.

Figure 9 Effect of resting time on static yield stress of cement pastes with 0 and $0.5 \%$ of nanoclay.

Figure 10 Effect of nanoclay addition on the critical strain of cement pastes, measured through low amplitude oscillatory sweep. 
Figure 1a

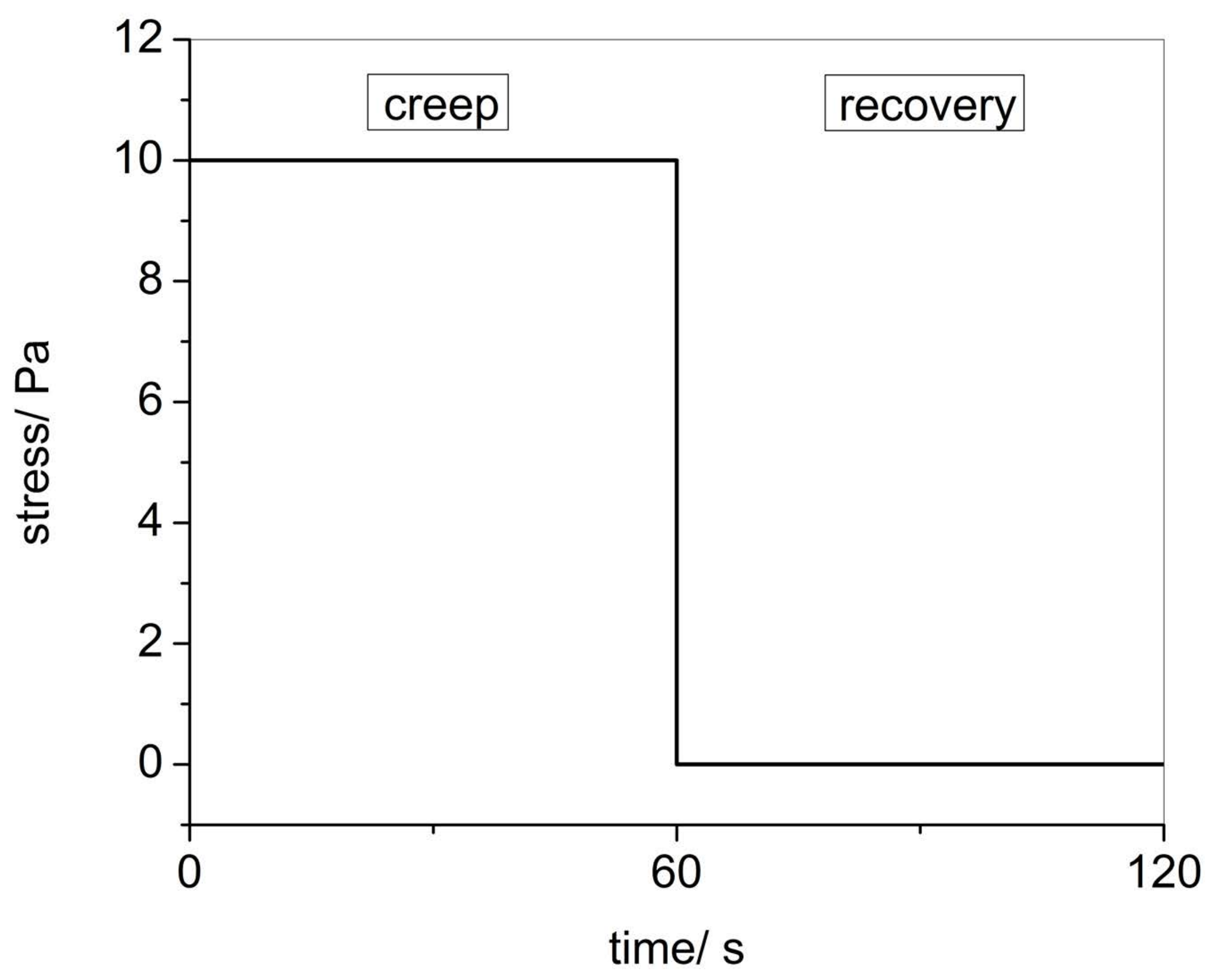


Figure 1b

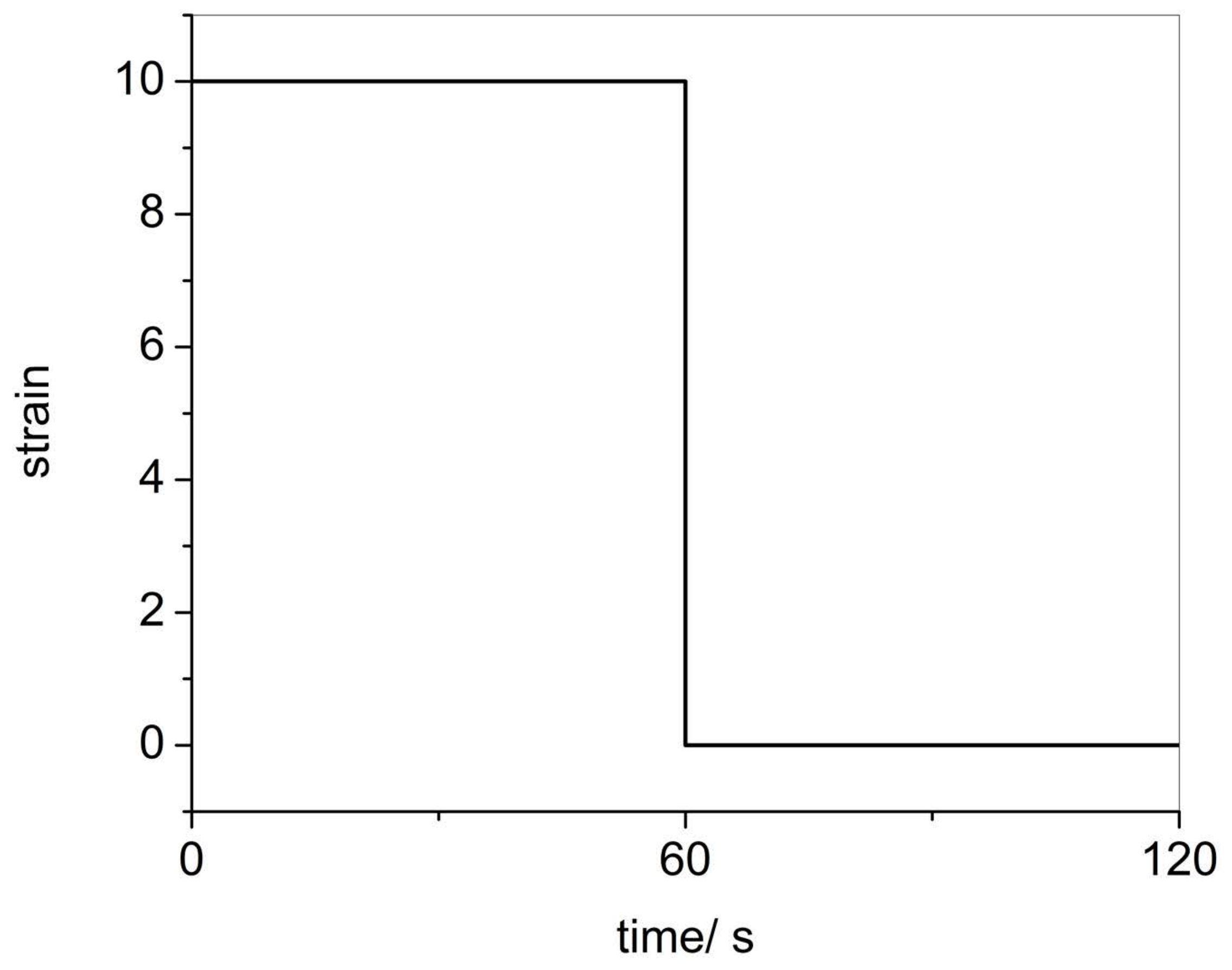


Figure 1c

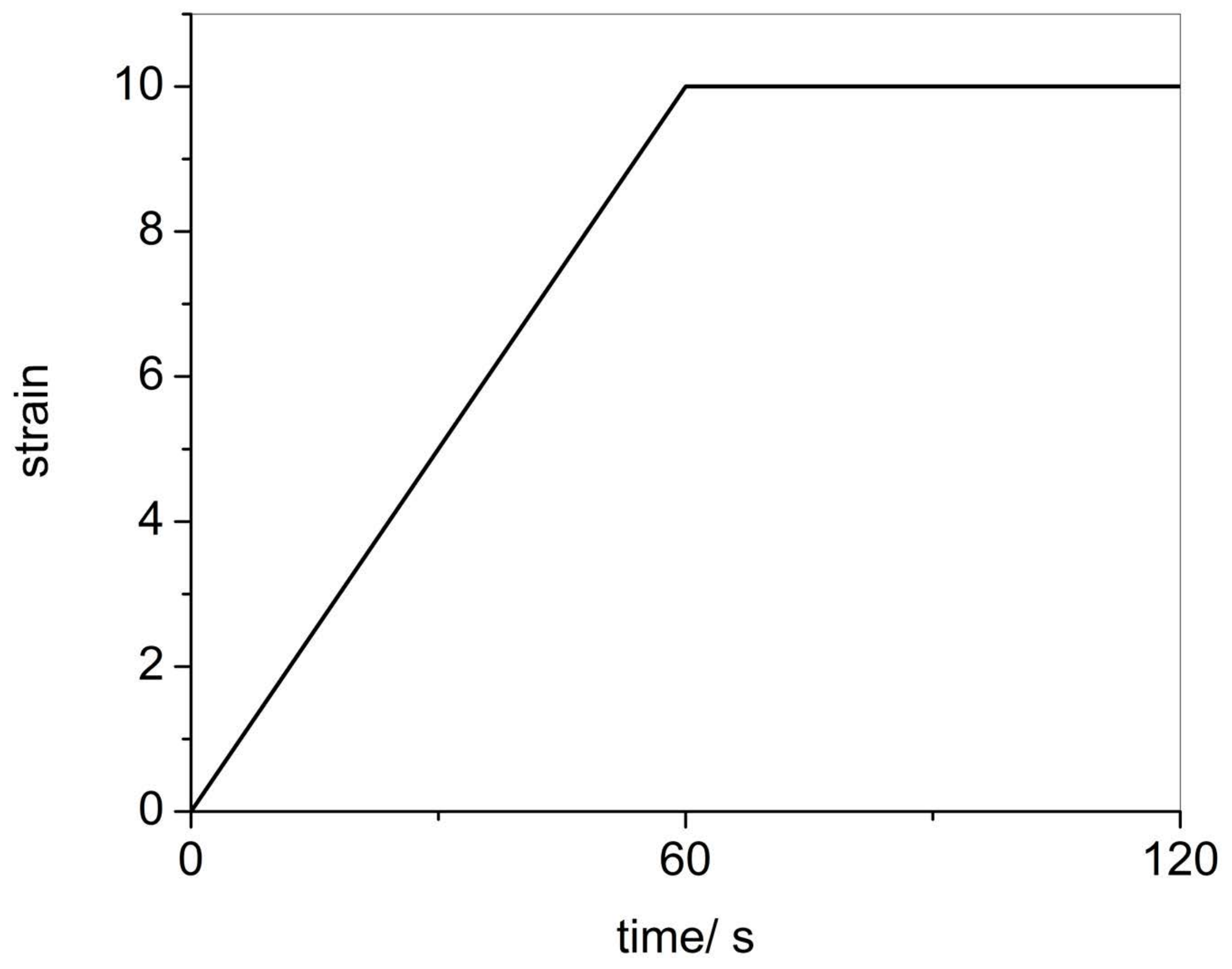


Figure 1d

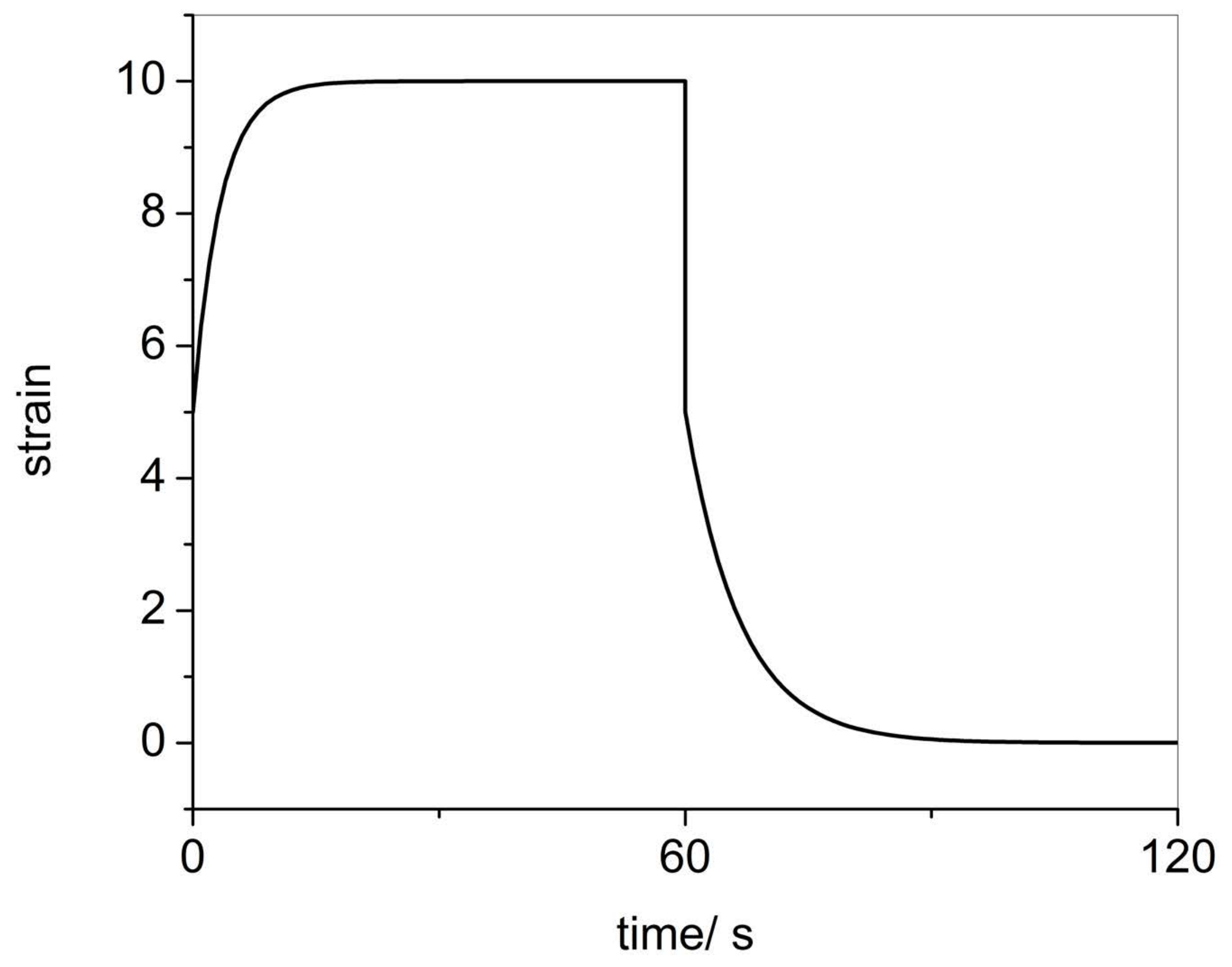


Figure 1e

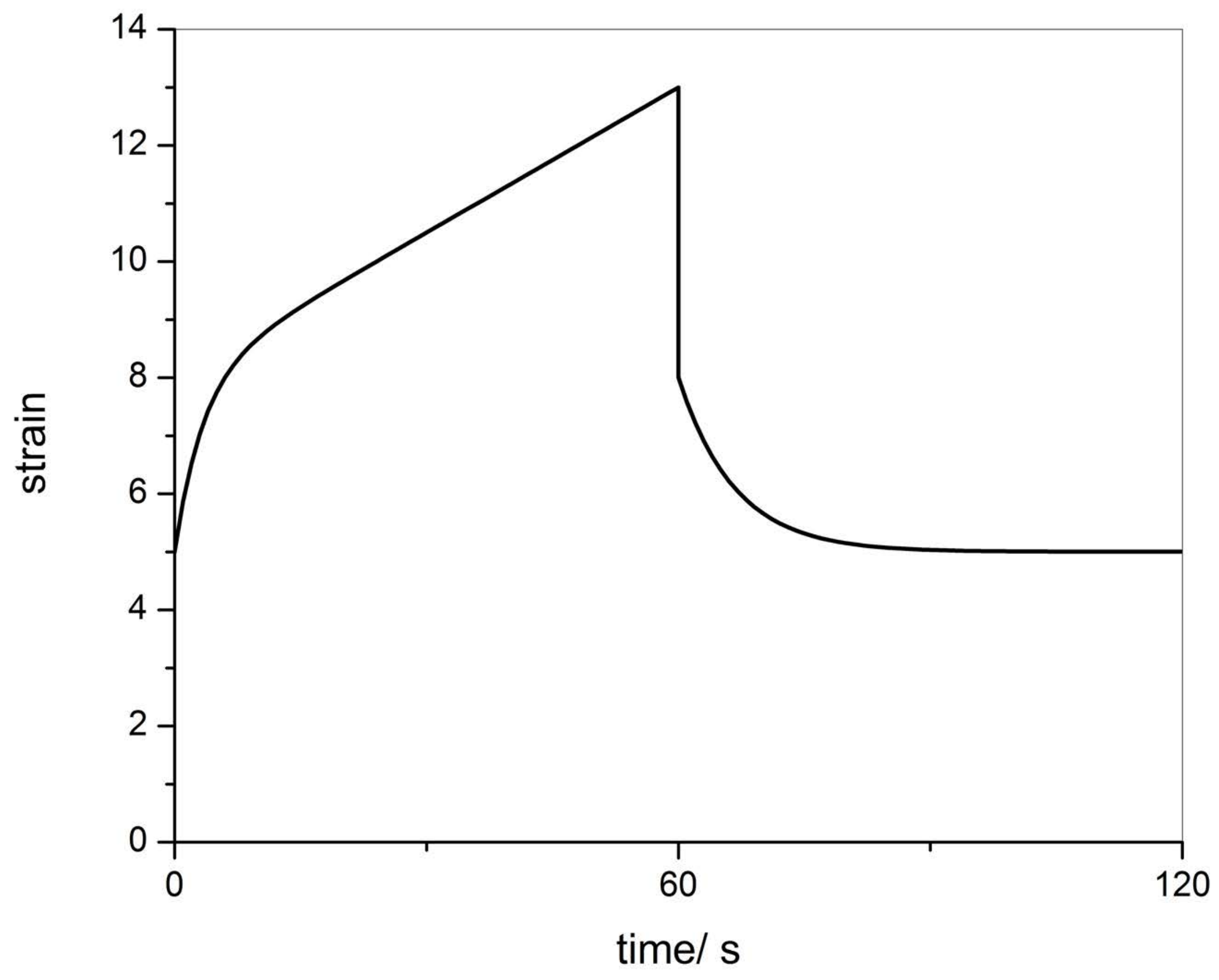


Figure 2

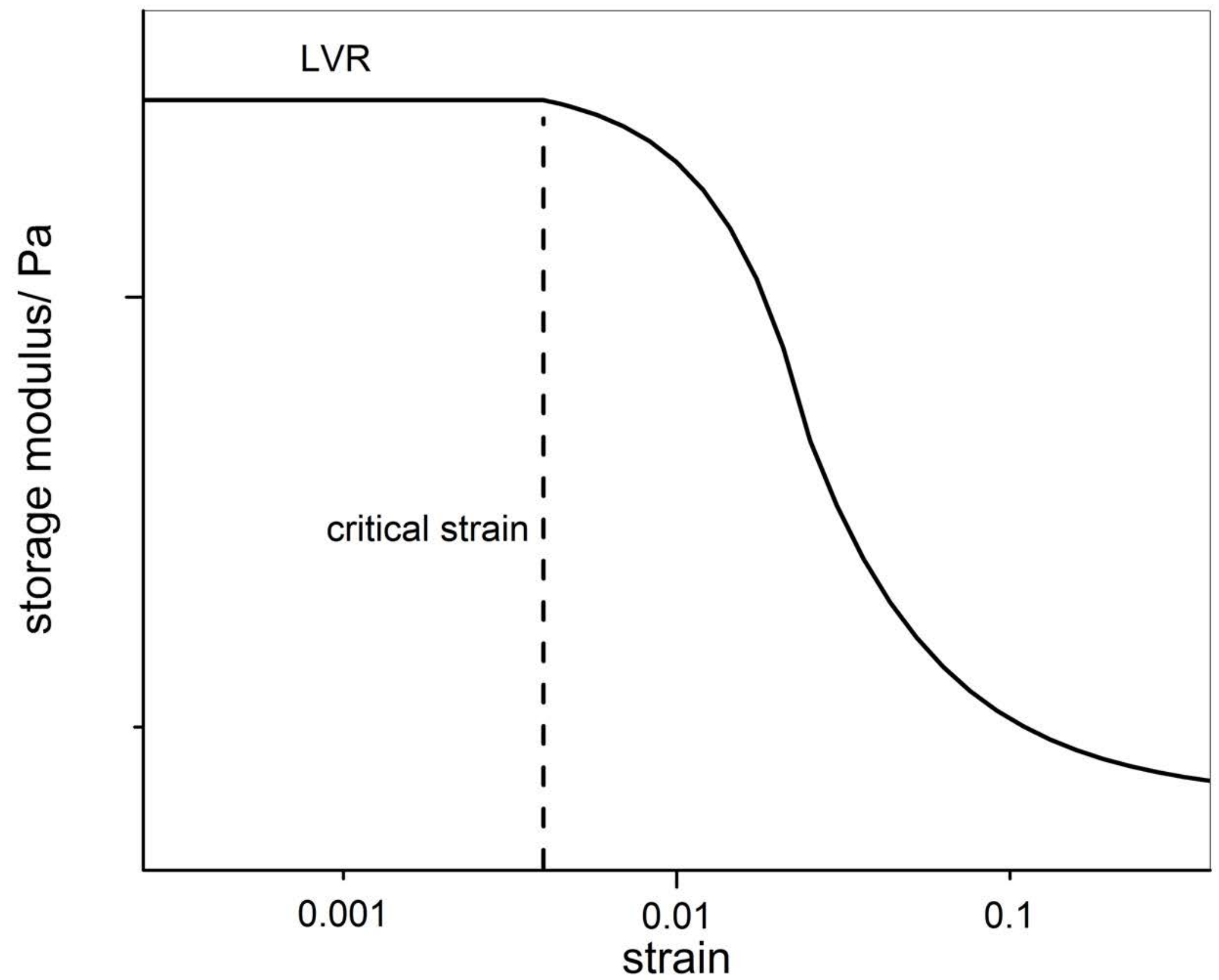


Figure $3 \mathrm{a}$

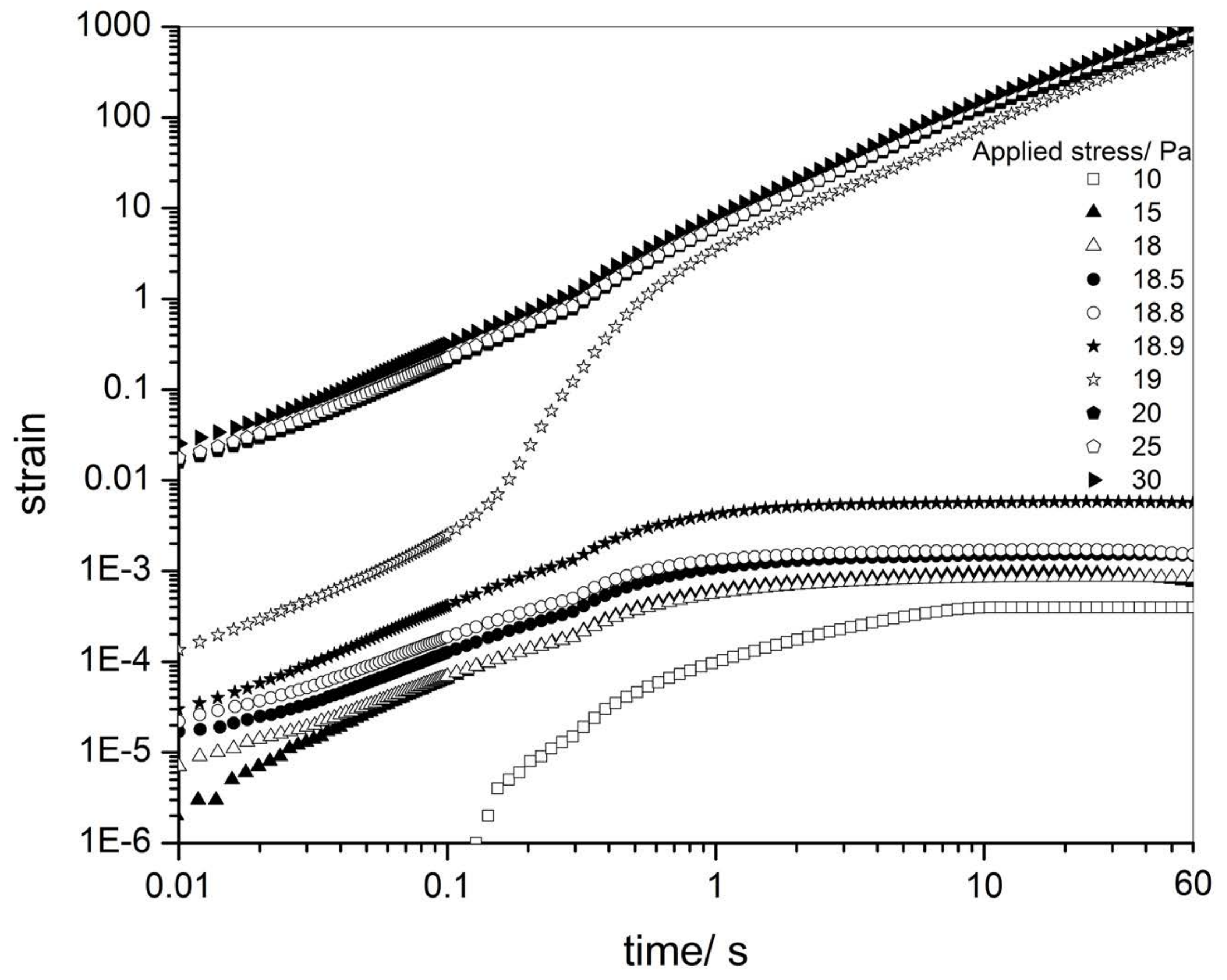


Figure $3 b$

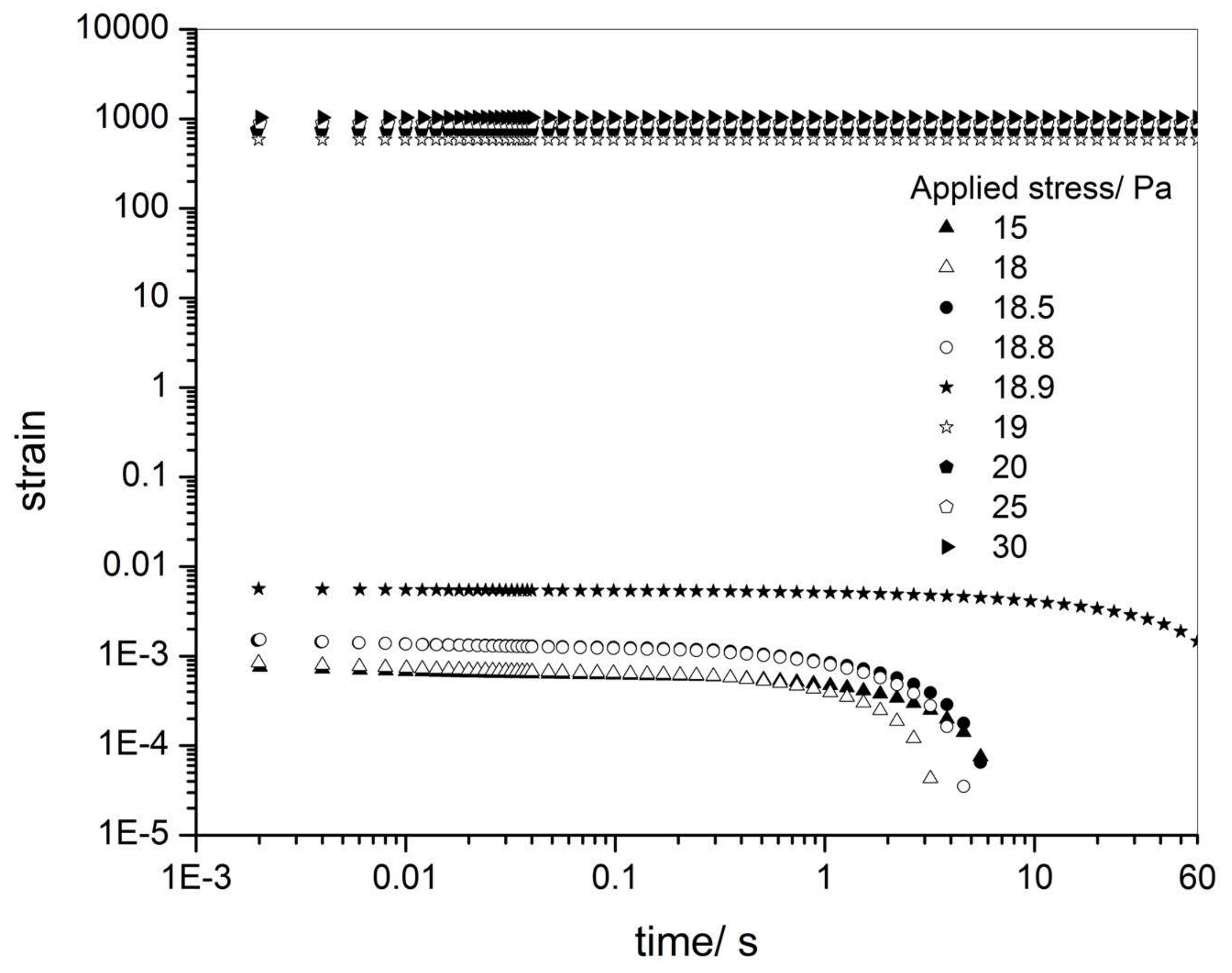


Figure 4

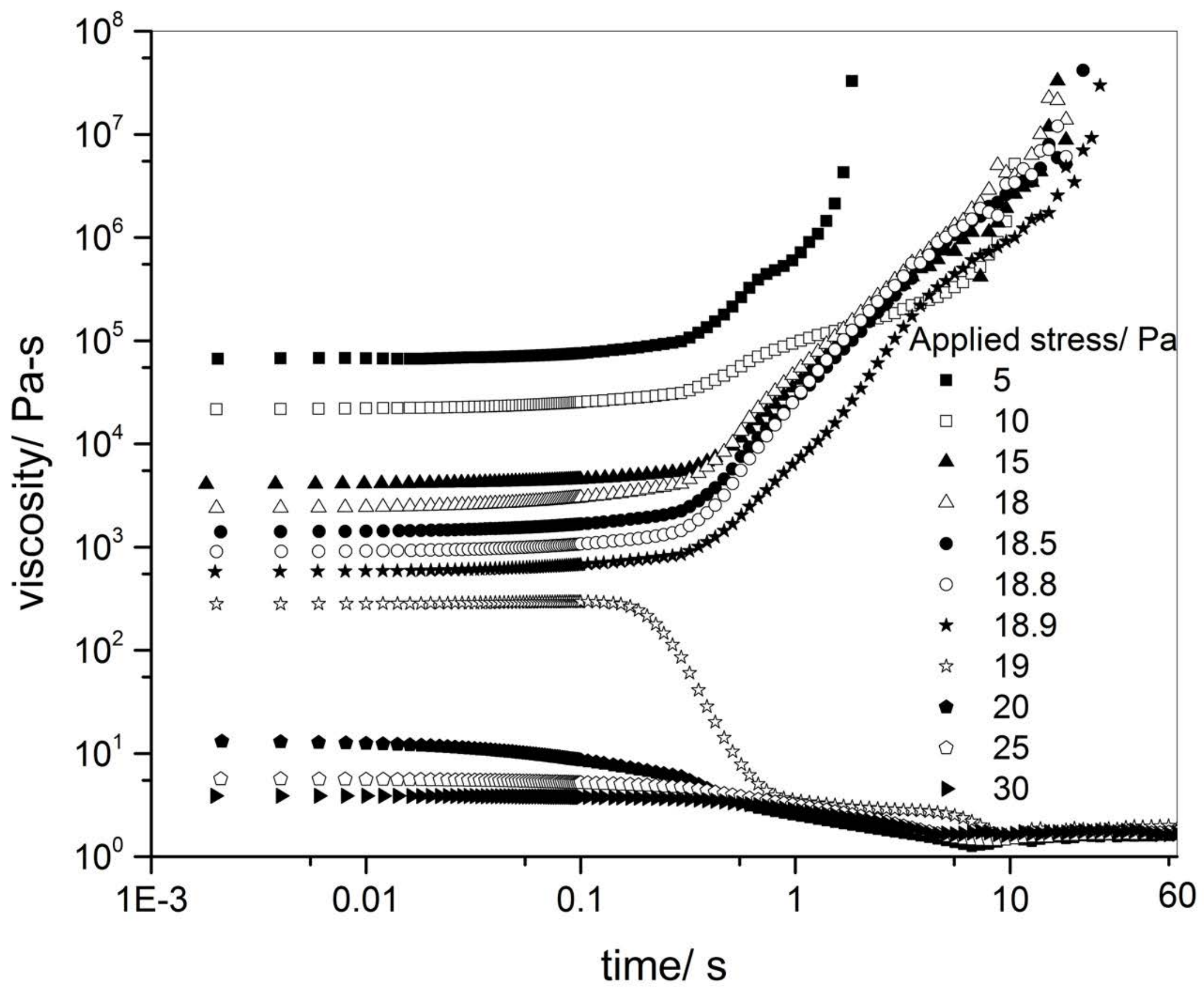


Figure 5

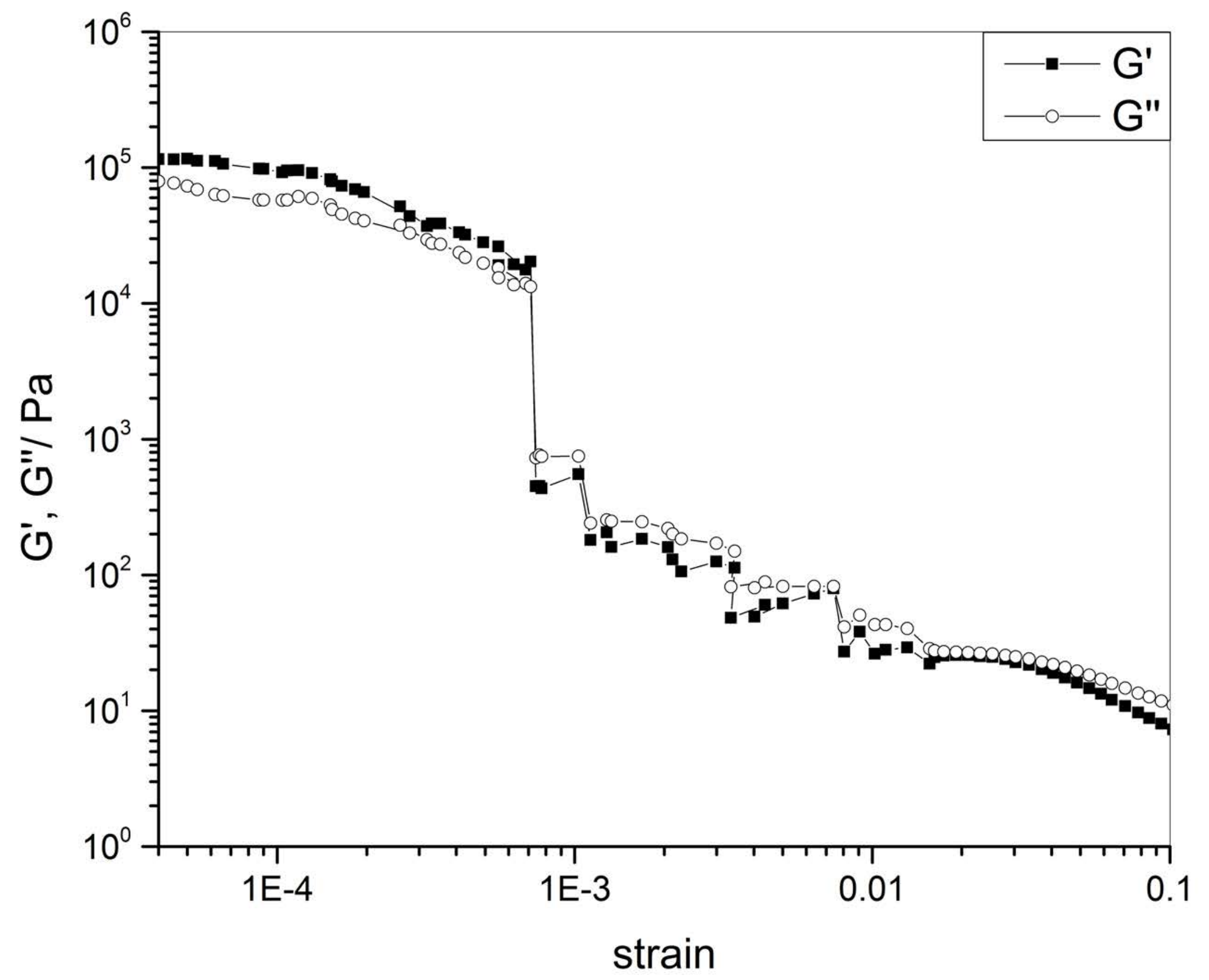


Figure 6a

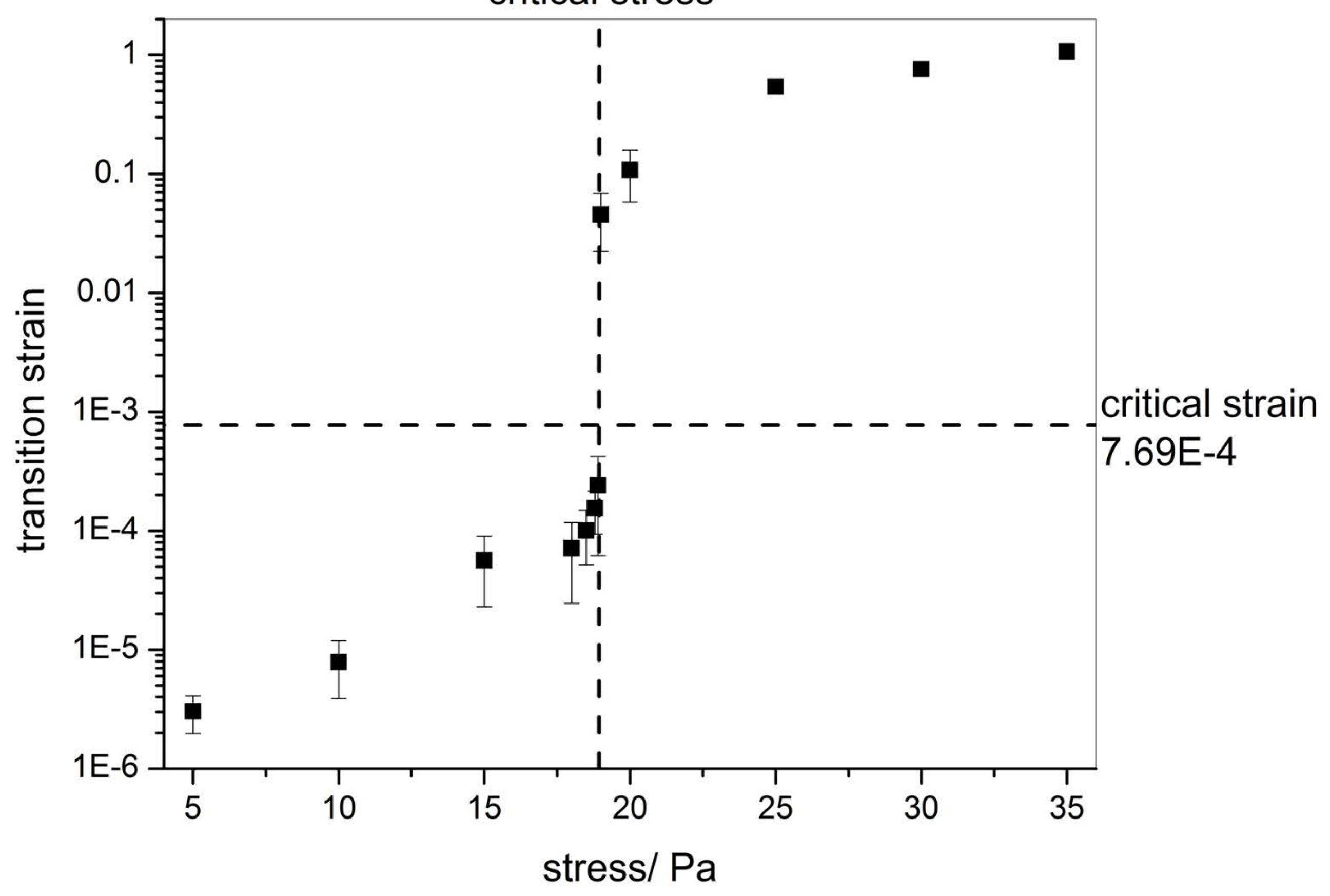


Figure $6 b$

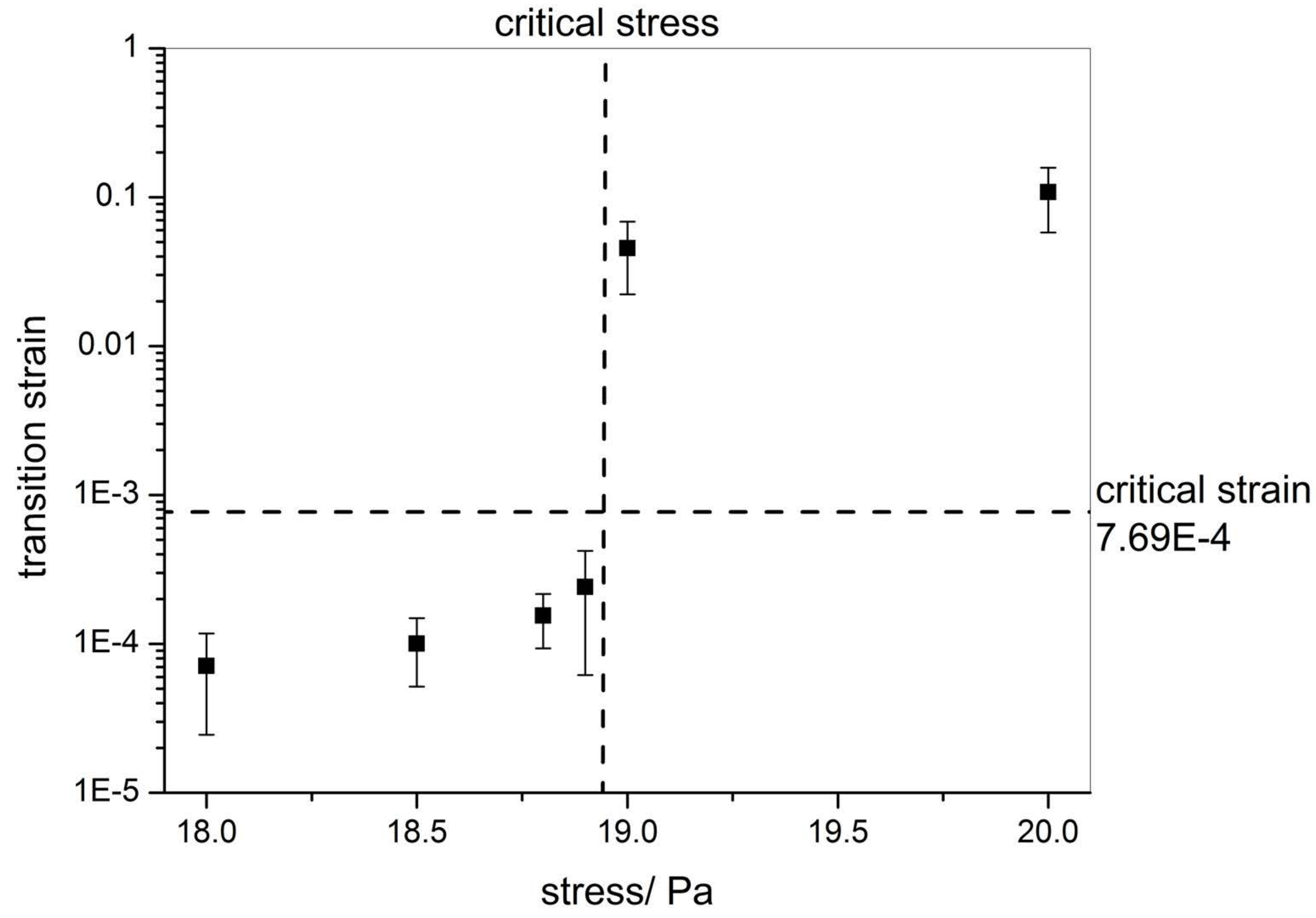


Figure 7
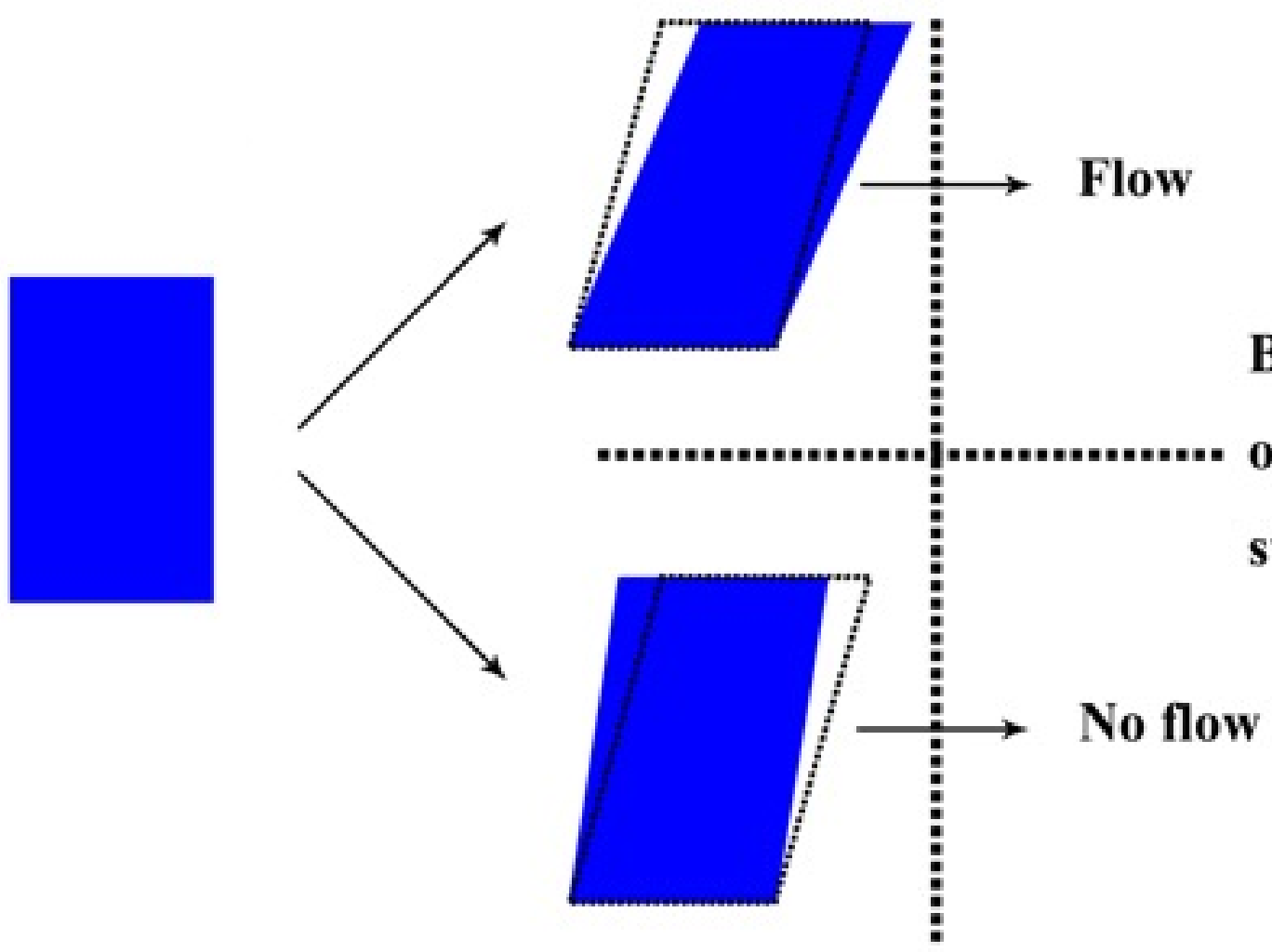

Bifurcation

occurs at static yield stress
Increasing applied stress

Transition point 
Figure 8

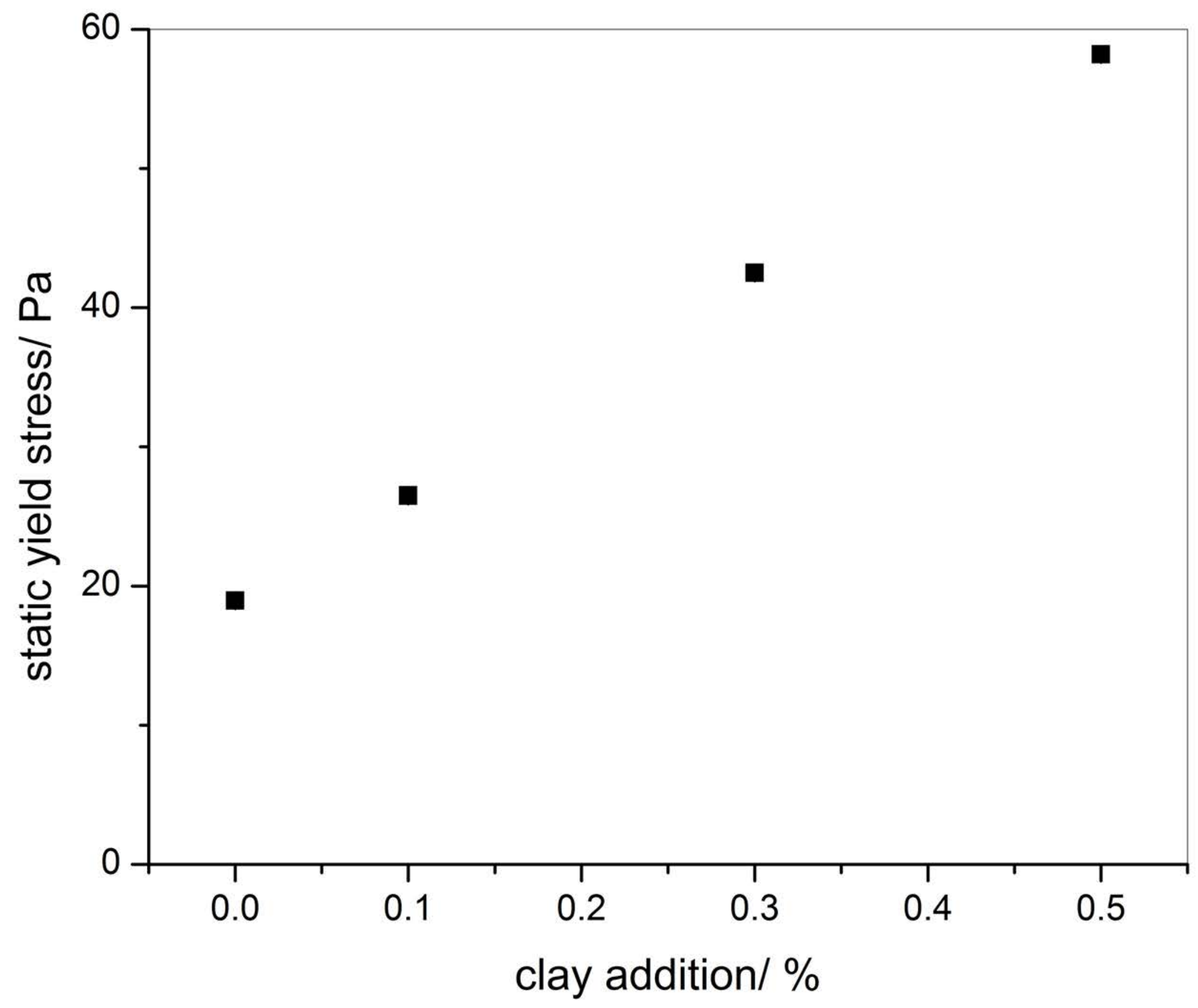


Figure 9

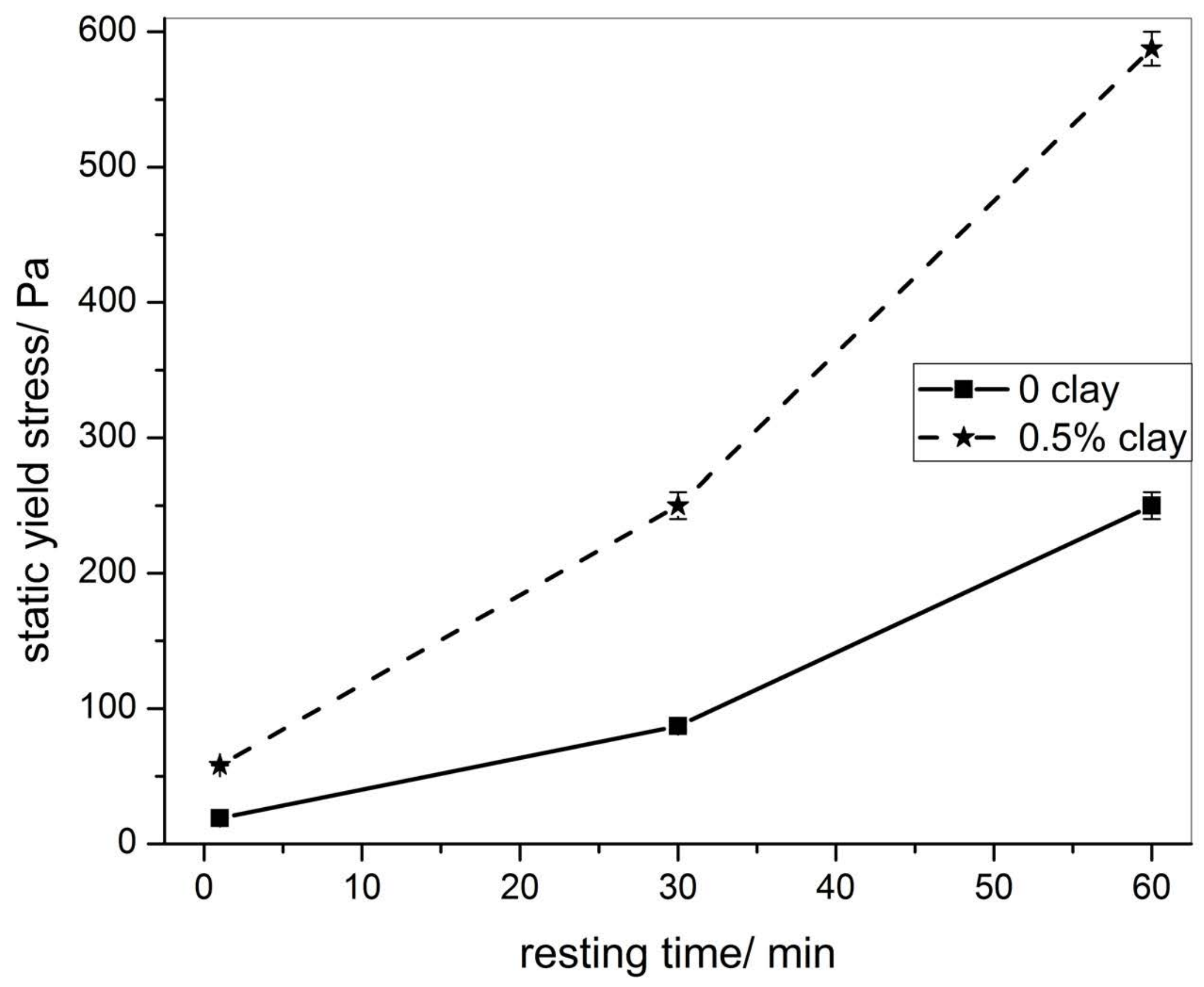


Figure 10

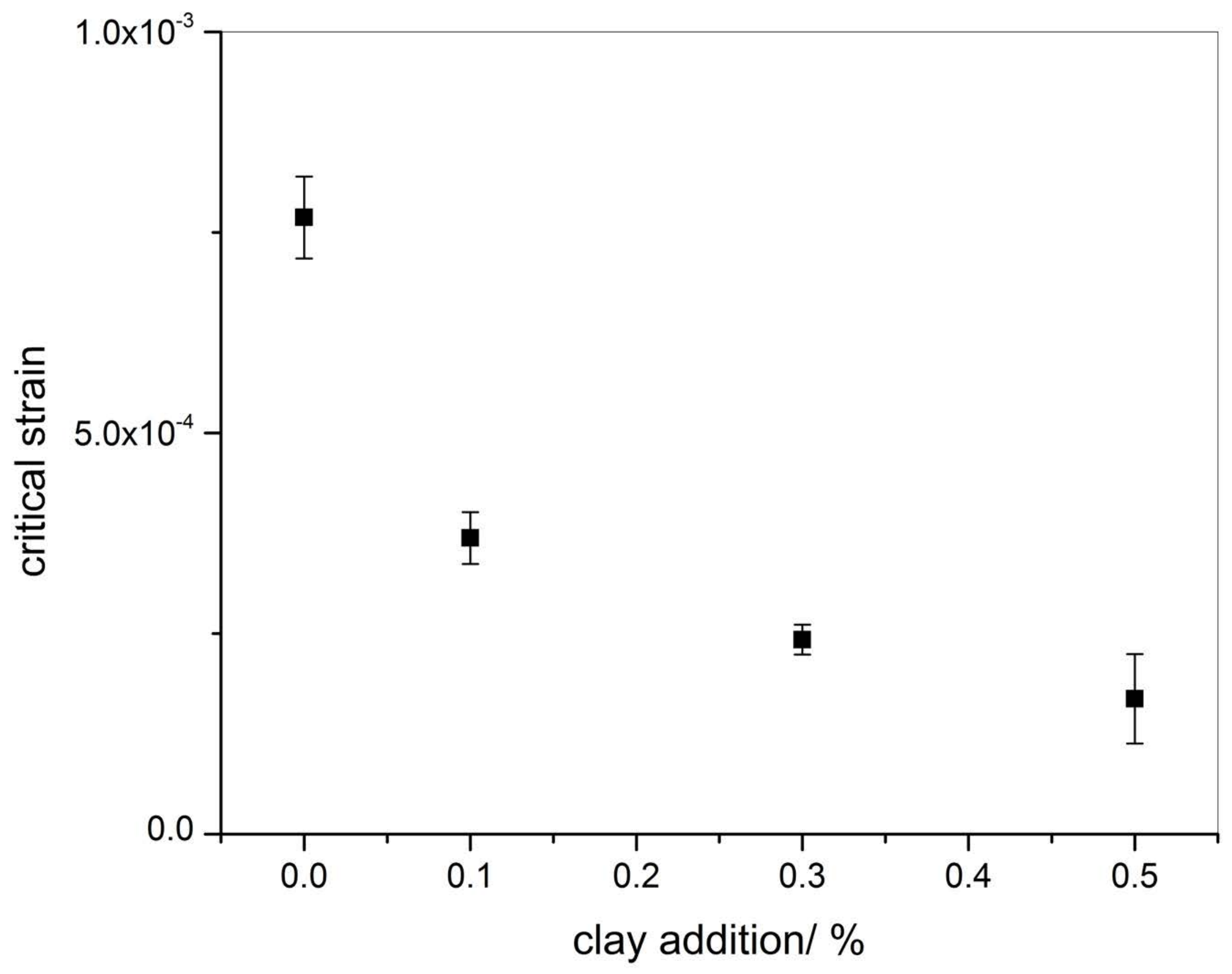

